\title{
A VO study of SDSS AGNs with X-ray emission from ROSAT-PSPC pointed observations
}

\author{
Robert J. Hanisch ${ }^{1}$, Anatoly A. Suchkov ${ }^{2}$, Timothy M. Heckman ${ }^{2}$, \\ and Wolfgang H. Voges ${ }^{3}$ \\ ${ }^{1}$ Space Telescope Science Institute, 3700 San Martin Drive,Baltimore, MD 21218, USA \\ email: hanisch@stsci.edu \\ ${ }^{2}$ Department of Physics and Astronomy, The Johns Hopkins University, \\ 3400 N. Charles St., Baltimore, MD 21218, USA \\ ${ }^{3}$ Max-Planck-Institut für extraterrestrische Physik, \\ Giessenbachstraße 1, D-85748 Garching, Germany
}

We use VO facilities to study AGNs with X-ray emission. We present a sample of 1744 of Type 1 AGNs from the Sloan Digital Sky Survey Data Release 4 (SDSS DR4) spectroscopic catalog with X-ray counterparts in the White-Giommi-Angelini catalogue (WGACAT) of ROSAT-PSPC pointed observations. Of 1744 X-ray sources, 1410 (80.9\%) are new AGN identifications. Of 4,574 SDSS DR4 AGNs for which we found radio matches in the catalogue of radio sources from the Faint Images of the Radio Sky at Twenty (FIRST) cm survey, 224 turned up in our sample of SDSS X-ray AGN.

We illustrate the content of our catalogue and its potential for AGN studies by providing statistical relationships for the catalogue data. The potential of the morphological information is emphasized by confronting the statistics of optically resolved, mostly lowredshift AGNs with unresolved AGNs that occupy a much wider redshift range. The immediate properties of the catalogue objects include significant correlation of X-ray and optical fluxes, which is consistent with expectations.

Also expected is the decrease of X-ray flux toward higher redshifts. The X-ray-tooptical flux ratio for the unresolved AGNs exhibits a decline toward higher redshifts, in agreement with previous results. The resolved AGNs, however, display the opposite trend. The X-ray hardness ratio shows a downward trend with increasing low-energy $\mathrm{X}$-ray flux and no obvious dependence on redshift. At a given optical brightness, X-ray fluxes of radio-loud AGNs are on average higher than those of radio-quiet AGNs by a factor of 2 .

We conclude that:

- VO-enabled comparisons and classifications of multi-wavelength source catalogs provide insights into nature of AGNs.

- Statistical analyses confirm several previously known correlations, but with much larger samples.

- The relationship between x-ray flux and optical flux differs for low-z resolved AGN and unresolved AGN.

- The sample of X-ray AGNs can be extended to $i>19.1$ by using AGN candidates with only photometric data.

The full version of this talk is available at the SPS 3 conference website: <http://www.ivoa.net/pub/VOScienceIAUPrague/programme/sess2-hanisch.ppt>. 